Ann. Biol. anim. Bioch. Biophys., 1977, 17 (3 A), 283-298.

\title{
Le système protéolytique de Penicillium Roqueforti. IV. Propriétés d'une carboxypeptidase acide.
}

\author{
par J.-C. GRIPON \\ avec la collaboration fechnique de Michèle NARDI
}

Laboratoire de Biochimie Microbienne, I.N.R.A., 78350 Jouy-en-josas.

Summary. Profeolytic system of Penicillium roqueforti.

IV. Properties of an acid carboxypeptidase.

An exfracellular acid carboxypeptidase has been isolated from the culture medium of Penicillium roqueforti. The enzyme was purified by ammonium sulfate precipitation, filtration on Biogel P-100, chromatography on DEAE and CM-cellulose and filtration on Biogel P-150.

The molecular weight of the enzyme was estimated at 110000 daltons by gel filtration. The optimum $\mathrm{pH}$ is 3.5-4.0 for Z-Glu-Tyr hydrolysis. At $35^{\circ} \mathrm{C}$ the enzyme is stable in the range 5.0-5.5; optimum temperature is $40^{\circ} \mathrm{C}$. The enzyme was insensitive to metal cations and was not inhibited by metal chelators or sulfhyldryl reagents (PHMB, MIAA). DFP inhibited enzyme activity but $\mathrm{pH}$ dependency was observed. Gly in the penultimate position or in C-terminal position caused poor hydrolysis of $\mathrm{N}$-substituted dipeptides. The action of the enzyme on glucagon showed that it is non-specific and releases neutral, basic or acid amino acids. However, the enzyme cleaved the $\mathrm{PrO}_{28}{ }^{-} \mathrm{Lys}_{29}$ peptide bond of the oxydized insulin B-chain very slowly.

The properties of this enzyme were similar to those of other acid carboxypeptidases from molds.

\section{Introduction.}

Les nombreuses études réalisées sur le système protéolytique exocellulaire des moisissures ont permis de montrer la complexité de ce dernier. En dehors des activités endopeptidasiques du type protéases acides (Fukumoto, Tsuru et Yamamoto, 1967 ; Hofmann et Shaw, 1964 ; Yu ef al., 1968), métalloprotéases (Nakasai, Nasuno ef Iguchi, $1973 b$; Sekine, 1972), protéases à groupe sérine (Turkova ef al., 1969 ; Hayashi, Fukushima et Noji, 1967), des activités exopeptidasiques ont été mises en évidence. En particulier une activité carboxypeptidasique à $\mathrm{pH}$ acide a été détectée ef des carboxypeptidases acides ont été étudiées pour une levure (Hayashi, Moore et Stein, 1973a) et certaines moisissures (Nakadai, Nasuno et Igushi, 1972a; Jones et Hofmann, 1972 ; Ichishima, 1972). II est maintenant démontré que ces enzymes diffèrent nettement des carboxypeptidases des tissus animaux : en dehors de leur $\mathrm{pH}$ d'action très différent, 
elles ne sont pas inhibées par les agents chélateurs des métaux mais par un réactif de la sérine le DFP. De plus leur spécificité est large puisqu'elles libèrent les acides aminés C-terminaux apolaires, acides ou basiques, y compris pour certaines (Hayashi, Moore ef Stein, 1973a ; Arai et Ichishima, 1974; Hui ef al., 1974) la proline. Des enzymes présentant des caractères comparables ont été mises en évidence dans des organes végétaux tels que la graine de l'orge (Visuki, Mikoba et Enari, 1969) ou l'exocarpe de certains agrumes (Kubota et al., 1973 ; Sprossler, 1971).

En ce qui concerne le système protéolytique exocellulaire de Penicillium roqueforti on a constaté qu'il comportait des endopeptidases (une protéase acide (Zevaco, Hermier et Gripon, 1973) ef une protéase neutre (Gripon ef Hermier, 1974)) mais également des activités aminopeptidasiques et carboxypeptidasiques. Dans le présent mémoire on se consacrera uniquement à l'étude de l'activité carboxypeptidasique à $\mathrm{pH}$ acide ef l'on constatera que cette enzyme a des propriétés analogues à celles des carboxypaptidases de moisissures déjà étudiées.

\section{Techniques expérimentales et matériel utilisé.}

1) Production de l'enzyme.

La souche utilisée ainsi que les conditions de culture ont été précédemment décrites (Zevaco, Hermier et Gripon, 1973). Le filtrat de culture était recueilli après $72 \mathrm{~h}$ d'incubation alors que le $\mathrm{pH}$ du milieu était de 4,5 .

\section{2) Mesure de l'activité carboxypeptidasique.}

L'activité était mesurée sur le Z-Glu-Tyr (Sigma) par la mesure de la quantité de tyrosine libérée à l'aide de la ninhydrine. $0,1 \mathrm{ml}$ de solution enzymatique en tampon acétate $0,1 \mathrm{M} \mathrm{pH} \mathrm{4,0} \mathrm{était} \mathrm{ajouté} \mathrm{à} 0,9 \mathrm{ml}$ de Z-Glu-Tyr $0,810^{-3} \mathrm{M}$ dans le même tampon. Après $10 \mathrm{mn}$ d'incubation à $35^{\circ} \mathrm{C}$ la réaction était arrêtée par l'addition de $1 \mathrm{ml}$ de ninhydrine préparée selon Moore et Stein (1954) et le mélange était immédiatement placé dans un bain-marie bouillant pendant exactement $15 \mathrm{mn}$, puis dans un bain d'eau froide pendant $5 \mathrm{mn}$. Après addition de $8 \mathrm{ml}$ d'éthanol à $50 \mathrm{p} .100$ la densité optique de la solution était lue à $570 \mathrm{~nm}$. Une unité carboxypeptidasique est définie comme la quantité d'enzyme nécessaire à la libération d'une micromole de tyrosine par minute dans les conditions précédentes.

\section{3) Dosage des protéines.}

Les protéines sont dosées selon la méthode de Lowry (1951) avec le réactif de Folin et Ciocalteu. La sérum-albumine a servi de protéine étalon.

\footnotetext{
Abréviations : $C M$ : carboxyméthyle ; DEAE : diéthylaminoéthyle ; PMC : chlorure phényle mercurique; $M M B$ : bromure méthyle mercurique ; PHMB : p-hydroxymercuribenzoate ; MIAA : acide monoiodoacétique ; TPCK : p-tosyl-L-phénylalanine chlorométhylcétone ; TLCK : N- $\alpha$-p-tosyl-Llysine chlorométhylcétone; DFP : diisopropyle fluorophosphate ; Z-Glu-Tyr : N-carbobenzoxy-Lglutamyl-L-tyrosine.
} 
4) Action sur les substrats synthétiques.

Ils ont été soumis à l'action de la carboxypeptidase à une concentration de $0,510^{-3}$ en tampon acétate $0,1 \mathrm{M} \mathrm{pH} \mathrm{4,0.} \mathrm{En} \mathrm{cas} \mathrm{d'insolubilité} 1$ p. 100 de diméthylformamide était ajouté au tampon (il a été vérifié à l'aide du Z-Glu-Tyr que la présence de ce composé ne modifie pas la vitesse d'hydrolyse du substrat). $0,1 \mathrm{ml}$ de solution enzymatique était ajouté à $0,9 \mathrm{ml}$ de substrat. L'hydrolyse était détectée par coloration à la ninhydrine comme précédemment décrit.

\section{5) Action sur les chaînes polypeptidiques.}

La chaîne B de l'insuline (Mann), le glucagon (Calbiochem), l'hexapeptide HisSer-GIn-Gly-Thr-Phe (Mann) étaient incubés en tampon acétate $0,02 \mathrm{M}$ (pH réactionnel : 5,5) à des concentrations respectives de 1,60,1,60 et $0,66 \mathrm{mg} / \mathrm{ml}$. Le rapport pondéral enzyme-substrat était de 1/125. Les hydrolyses étaient suivies par prélèvement d'aliquotes, puis électrophorèse sur papier à $\mathrm{pH} 1,9$. Après 24 h à $30^{\circ} \mathrm{C}$ l'enzyme était inactivée par alcalinisation du milieu et le mélange analysé sur un autoanalyseur Beckman Unichrom.

6) Détermination du poids moléculaire.

Le poids moléculaire a été estimé par filtration sur gel (Andrews, 1964) sur une colonne de Biogel P $150(100 \times 2,1 \mathrm{~cm})$ équilibrée en tampon acétate $0,1 \mathrm{M} \mathrm{PH} \mathrm{5,5}$. L'aldolase du muscle de lapin (PM: 158000 daltons), la sérum-albumine bovine (PM : 67000 daltons), l'albumine de l'œuf (PM : 45000 daltons) et le chymotrypsinogène (PM 25000 daltons) ont servi de protéines étalons.

\section{7) Electrophorèses.}

Les électrophorèses étaient réalisées soit en plaques d'acrylamide agarose, soit en tube d'acrylamide. En acrylamide agarose le tampon était un tampon Tris-glycine $\mathrm{pH}$ 8,7, préparé selon Uriel (1966). Les protéines étaient colorées par immersion $\epsilon \dagger$ agitation pendant $3 \mathrm{~h}$ dans le bleu de Coomassie $(0,012 \mathrm{p} .100)$ en solution dans le mélange eau, méthanol, acide acétique, glycérol $(52,5 ; 40 ; 5 ; 2,5)$. La décoloration avait lieu pendant une nuit dans une solution à 40 p. 100 de méthanol contenant 5 p. 100 d'acide acétique et 3 p. 100 de glycérol. Les électrophorèses en tube d'acrylamide étaient effectuées en tampon Tris-borate $0,1 \mathrm{M} \mathrm{pH} \mathrm{9,2} \mathrm{ou} \mathrm{en} \mathrm{tampon} \beta$ alanine-acide acétique $\mathrm{pH} 4,3$ (Reisfeld, Lewis et Williams, 1962). Le bleu de Coomassie utilisé selon la méthode de Chrambach ef al. (1967) a été employé pour la révélation des protéines.

L'existence de plusieurs protomères dans la molécule enzymatique a été testée par électrophorèse en gel d'acrylamide en présence de SDS selon la méthode de Dunker et Rueckert (1969).

8) Purification de l'enzyme.

Le mycélium éłait séparé du milieu de culture par filtration sous vide sur filtre Durieux $n^{\circ} 111$. Les spores et débris mycéliens restant en suspension étaient éliminés par centrifugation en continu à $27000 \mathrm{~g}$ et $4{ }^{\circ} \mathrm{C}$. 
La concentration des protéines avaif lieu par précipitation au sulfate d'ammonium à $80 \mathrm{p}$. 100 de saturation. Après un séjour d'une semaine à $4^{\circ} \mathrm{C}$ le précipité était récupéré par filtration sur filtre en fibre de verre (Watman $G F / F)$ puis mis en solution dans un tampon phosphate $0,1 \mathrm{M} \mathrm{pH} \mathrm{6,0}$.

- Filtration sur Bio-Gel P-100.

Après concentration préalable sur membrane Diaflo UM 10 les protéines étaient chromatographiées sur une colonne de Bio-Gel P-100 $(4,2 \times 132 \mathrm{~cm})$ en tampon phosphate $0,1 \mathrm{M} \mathrm{pH} \mathrm{6,0}$. Le volume de l'échantillon était inférieur ou égal à $80 \mathrm{ml}$. Les fractions actives étaient regroupées ef dialysées pendant une nuit contre du tampon phosphate $0,01 \mathrm{M} \mathrm{pH} 6,0$.

- Chromatographie sur DEAE-cellulose.

Par fractions d'environ $20 \mathrm{mg}$ l'extrait enzymatique était chromatographié sur une colonne de DEAE-cellulose $(2,5 \times 10,5 \mathrm{~cm})$ équilibrée en tampon phosphate $0,01 \mathrm{M} \mathrm{pH} 6,0$ et élué par un gradient de même tampon de molarité 0,01-0,2 M.

- Chromatographie sur CM-cellulose.

Après regroupement et dialyse contre un tampon phosphate $0,01 \mathrm{M} \mathrm{pH} 6,0$ les protéines actives sont placées par fractions de $10 \mathrm{mg}$ ou moins au sommet d'une colonne de $C M$-cellulose $(2,5 \times 10 \mathrm{~cm})$ équilibrée avec le même tampon. Les protéines fixées sont éluées par un gradient de tampon phosphate 0,01-0,35 M.

- Filtration sur Bio-Gel P-150.

La fraction active non fixée sur $C M$-cellulose est concentrée sur membrane Diaflo UM 20, puis chromatographiée par volume de $5 \mathrm{ml}$ sur colonne de Bio-Gel P-150 $(2,0 \times 99 \mathrm{~cm})$ équilibrée en tampon acétate $0,1 \mathrm{M} \mathrm{pH} 5,5$.

\section{Résultats.}

\section{Purification (fig. 1, 2, 3 et 4).}

Les pigments encore présents en grande quantité après la précipitation au sulfate d'ammonium sont éliminés sur Bio-Gel P-100 (fig. 1). Par chromatographie sur DEAE-cellulose (fig. 2) une seule activité carboxypeptidasique est éluée à une molarifé de $0,10 \mathrm{M}$. Sur $\mathrm{CM}$-cellulose (fig. 3) les protéines actives sont séparées en une fraction non fixée sur la colonne représentant environ 95 p. 100 de l'activité totale et une fraction mineure fixée sur la colonne. Cette dernière fraction (fraction $B$ ) a été mise de côté pour comparaison ultérieure avec la préparation purifiée. La purification a été poursuivie sur les protéines non fixées par une chromatographie sur BioGel P-150 (fig. 4) qui élimine une quantité notable des protéines de l'extrait.

Un bilan de la purification est présenté dans le tableau 1. L'activité spécifique du filtrat de culture brut n'a pu être déterminée sur le Z-Glu-Tyr à cause de la présence des groupements aminés de l'hydrolysat trypsique de caséine composant le milieu. 
TABLEAU 1

Bilan de purification

\begin{tabular}{|c|c|c|c|c|c|c|}
\hline & $\begin{array}{l}\text { Protéines } \\
(\mathrm{mg})\end{array}$ & $\begin{array}{l}\text { Volume } \\
\text { (ml) }\end{array}$ & Unités & $\begin{array}{l}\text { Activité } \\
\text { spécifique } \\
(\mathrm{U} / \mathrm{mg})\end{array}$ & $\begin{array}{l}\text { Rendement } \\
\text { (p. 100) }\end{array}$ & Purification \\
\hline $\begin{array}{c}\text { Filtrat de culture } \ldots \ldots . \\
\text { Précipité au sulfate } \\
\text { d'ammonium } \ldots \ldots \ldots \\
\text { Bio-gel P-100....... } \\
\text { DEAE-cellulose } \ldots \ldots \ldots \\
\text { CM-cellulose } \ldots \ldots \ldots \ldots \\
\text { Bio-gel P-150....... }\end{array}$ & $\begin{array}{c}13084 \\
2145 \\
92 \\
34 \\
15 \\
3,75\end{array}$ & $\begin{array}{r}18000 \\
\\
165 \\
285 \\
186 \\
150 \\
84\end{array}$ & $\begin{array}{r}111,3 \\
96,8 \\
73,5 \\
48,9 \\
35,2\end{array}$ & $\begin{array}{l}0,05 \\
1,05 \\
2,16 \\
3,26 \\
9,38\end{array}$ & $\begin{array}{r}100 \\
87 \\
66 \\
44 \\
32\end{array}$ & $\begin{array}{r}1 \\
20 \\
42 \\
63 \\
183\end{array}$ \\
\hline
\end{tabular}

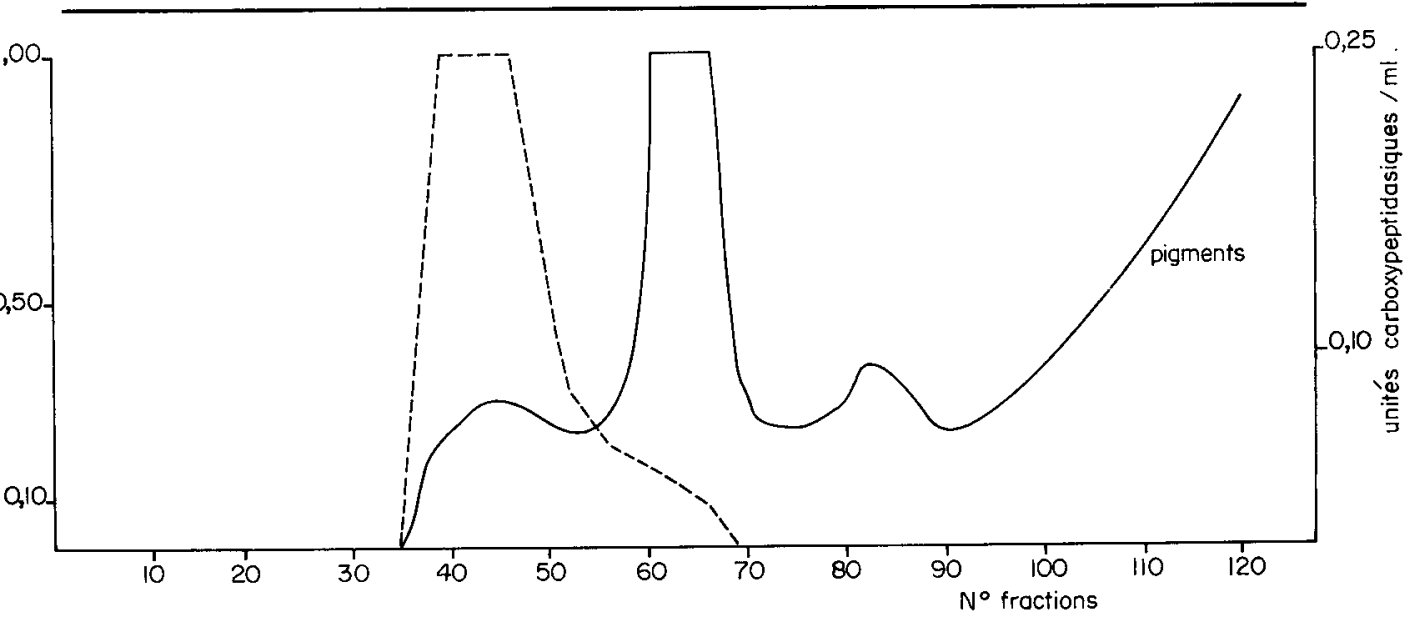

FIG. 1. - Chromatographie sur Bio-gel P-100

Colonne : $132 \times 4,2 \mathrm{~cm}$; Volume d'échantillon : $80 \mathrm{ml}$; Débit : $60 \mathrm{ml} / \mathrm{h}$; Fractions : $12 \mathrm{ml}$; Elution en tampon phosphate $0,1 \mathrm{M} \mathrm{pH} 6,0$.

_ : Densité optique à $280 \mathrm{~nm}$. … - . : Activité carboxypeptidasique.

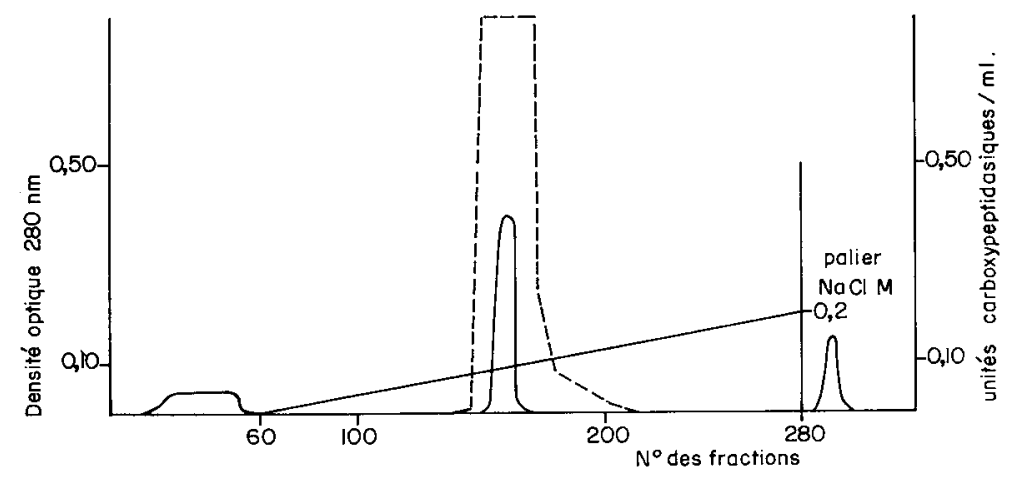

FIG. 2. - Chromatographie sur D.E.A.E.-cellulose.

Colonne : $10,5 \times 2,5 \mathrm{~cm}$; Volume d'échantillon : $180 \mathrm{ml}$; Débit : $30 \mathrm{ml} / \mathrm{h}$; Fractions : $5 \mathrm{ml}$; Tampon phosphate $0,01 \mathrm{MPH} 6,0$; Gradient de tampon 0,01-0,2 $\mathrm{M}$ puis palier $\mathrm{NaCl} 1 \mathrm{M}$. : Densité optique à $280 \mathrm{~nm}$. $\quad$.....: Activité carboxypeptidasique. 


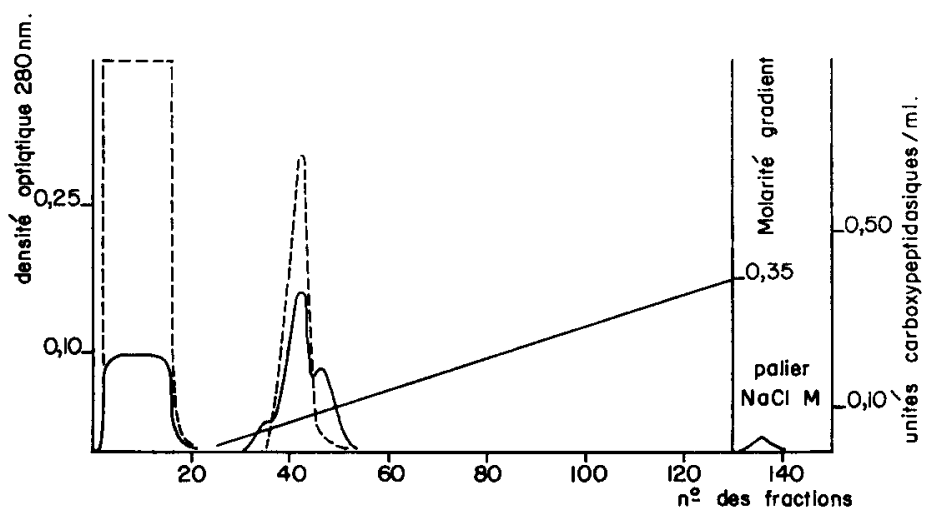

FIG. 3. - Chromatographie sur CM-cellulose.

Colonne : 2,5 $\times 10 \mathrm{~cm}$; Volume d'échantillon : $70 \mathrm{ml}$; Débit : $30 \mathrm{ml} / \mathrm{h}$; Fraction : $5 \mathrm{ml}$; Tampon phosphate $0,01 \mathrm{M} \mathrm{pH} \mathrm{6,0;} \mathrm{Gradient} \mathrm{de} \mathrm{tampon} \mathrm{0,01-0,35,} \mathrm{puis} \mathrm{palier} \mathrm{NaCl} 1 \mathrm{M}$.

- Densité optique à $280 \mathrm{~nm}$.

.... - : Activité carboxypeptidasique.

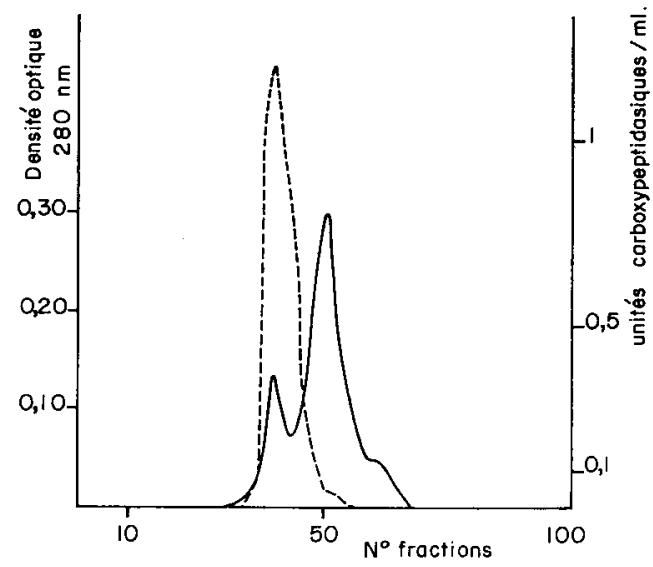

FIG. 4. - Chromatographie sur Bio-gel P-150.

Colonne : $99 \times 2,0 \mathrm{~cm}$; Volume d'échantillon : $5 \mathrm{ml}$; Débit : $10 \mathrm{ml} / \mathrm{h}$; Fractions : $2,5 \mathrm{ml}$; Elution en tampon acétate $0,1 \mathrm{M} \mathrm{pH} 5,5$.

- Densité optique à $280 \mathrm{~nm}$.

..... Activité carboxypeptidasique.

Le degré final de purification par rapport à la précipitation après sulfate d'ammonium est de 183. Il est à noter que la quantité initiale d'enzyme produite par $P$. roqueforti dans le milieu est faible (moins de $0,7 \mathrm{mg}$ par litre du milieu de culture).

Par électrophorèse de l'extrait enzymatique purifié en gel d'acrylamide à $\mathrm{pH}$ 4,3 une seule bande de protéine est obtenue. Par contre, à $\mathrm{pH}$ alcalin en gel d'acrylamide ou d'acrylamide agarose on observe la présence d'une bande principale ef d'une bande de coloration faible. La révélation de l'activité dans les gels d'acrylamide a été 
testée par découpage du gel en disques ef incubation de ces disques en présence de Z-Glu-Tyr tamponné à pH 4,0. Aucune activité n'a pu être détectée par cette méthode, l'enzyme étant probablement dénaturée par le $\mathrm{pH}$ alcalin du tampon d'électrophorèse. Cependant les courbes d'inactivation thermiques obtenues en exprimant le logarithme de l'activité résiduelle en fonction du temps de chauffage sont des droites (fig. 7) et indiquent qu'une seule activité carboxypeptidasique est présente dans la préparation. L'extrait enzymatique obtenu après P-150 a été considéré comme suffisamment purifié pour entreprendre l'élude de ses propriétés.

\section{Propriétés.}

1. Influence du $\mathrm{pH}$.

Le $\mathrm{pH}$ optimum d'action est de 3,5-4,0 sur le Z-Glu-Tyr en tampon citrate-phosphate (fig. 5). L'activité enzymatique est sensible à la nature du tampon présent ef à

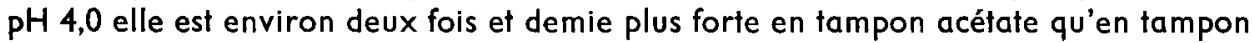
citrate-phosphate.

La stabilité vis-à-vis du $\mathrm{pH}$ a été mesurée entre $\mathrm{pH}$ 2,6 ef 7,0 après incubation en tampon citrate-phosphate $0,2 \mathrm{M}$ puis détermination de l'activité résiduelle sur le

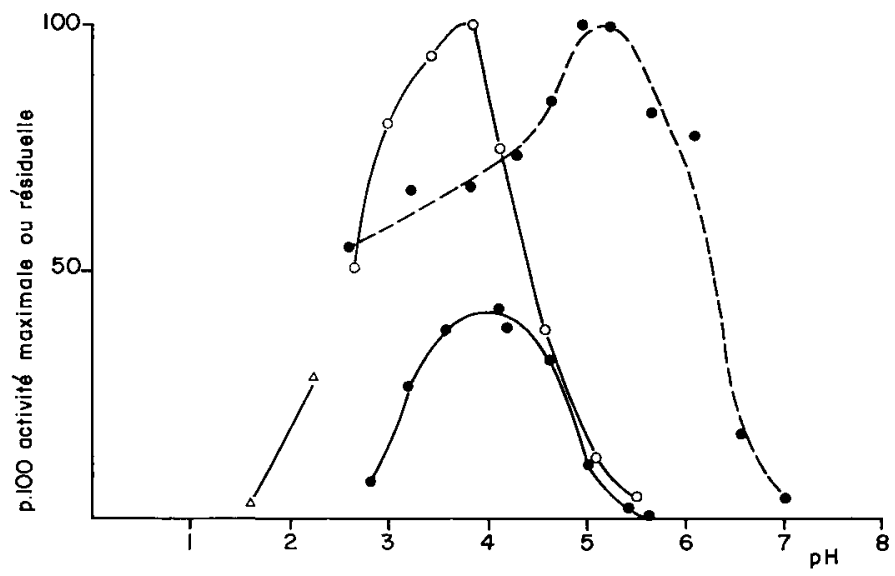

FIG. 5. - Influence du $\mathrm{pH}$.

a) $\mathrm{pH}$ optimum d'oction

$\triangle \longrightarrow \triangle$ : Tampon $\mathrm{KCl} \mathrm{HCl} 0,1 \mathrm{M}$

: Tampon citrate phosphate $0,1 \mathrm{M}$

O-_-O : Tampon acétate $0,1 \mathrm{M}$

Mélange d'hydrolyse : $-0,1 \mathrm{ml}$ d'enzyme en tampon acétate $\mathrm{pH} 5,50,05 \mathrm{M} ;-0,9 \mathrm{ml}$ de Z-GluTyr $0,8 \mathrm{mM}$ en tampon $0,1 \mathrm{M}$

Hydrolyse : $20 \mathrm{mn}$ à $35^{\circ} \mathrm{C}$.

b) Stabilité vis-d-vis du $\mathrm{pH}$

-.. : Tampon citrate phosphate

Incubation : $20 \mathrm{mn}$ à $35^{\circ} \mathrm{C}$ en tampon citrate phosphate $0,2 \mathrm{M}$.

Hydrolyse : selon matériel et méthodes. 
Z-Glu-Tyr tamponné à pH 4,0. Après $20 \mathrm{mn}$ à $35^{\circ} \mathrm{C}$ l'enzyme reste stable (fig. 5) dans une zone de $\mathrm{pH}$ étroite comprise entre 5,0 et 5,5. L'activité diminue très rapidement pour des pH supérieurs à 6,0 .

2. Influence de la température.

Sur le substrat Z-Glu-Tyr l'hydrolyse maximale est cbtenue à $40^{\circ} \mathrm{C}$ (fig. 6). En tampon acétate $0,1 \mathrm{M} \mathrm{pH} \mathrm{5,5}$ pendant $40 \mathrm{mn}$ à différentes températures l'enzyme reste stable jusqu'à $42^{\circ} \mathrm{C}$. La stabilité thermique diminue rapidement au-dessus de cette valeur, après $20 \mathrm{mn}$ d'incubation à $50^{\circ} \mathrm{C}$ on ne retrouve plus d'activité (fig. 7).

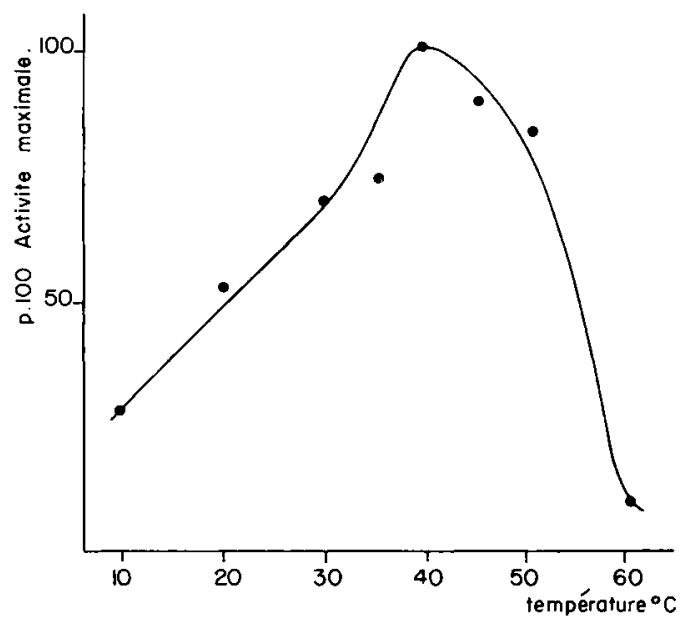

FIG. 6. - Température optimale d'action.

Mélange d'hydrolyse : $0,1 \mathrm{ml}$ de solution enzymatique en tampon acétate $0,1 \mathrm{MpH} 5,5 ;-0,9 \mathrm{ml}$ de Z-Glu-Tyr, 0,8 $\mathrm{mM}$ en tampon acétate $0,1 \mathrm{M}$ pH 4,0.

Incubation pendant $20 \mathrm{mn}$ à $35^{\circ} \mathrm{C}$.

3. Estimation du poids moléculaire.

Le poids moléculaire estimé par filtration sur Bio-Gel P-150 (fig. 8) est de 110000 daltons.

L'électrophorèse en présence de SDS ne permet pas de montrer l'existence de sousunités. En effet on observe, comme pour les électrophorèses à $\mathrm{pH}$ alcalin précédentes, l'existence d'une bande de protéine principale et d'une bande de faible intensité qui correspondent respectivement à des poids moléculaires de 102000 ef 115000 . La différence entre ces deux poids moléculaires semble trop forte pour admettre que ces deux bandes correspondent à des isoenzymes.

4. Action des inhibiteurs (tabl. 2).

Les agents chélateurs tels que l'EDTA ou l'O-phénantroline sont sans effet sur l'activité enzymatique. II en est de même des composés réducteurs comme le $\beta$-mer- 


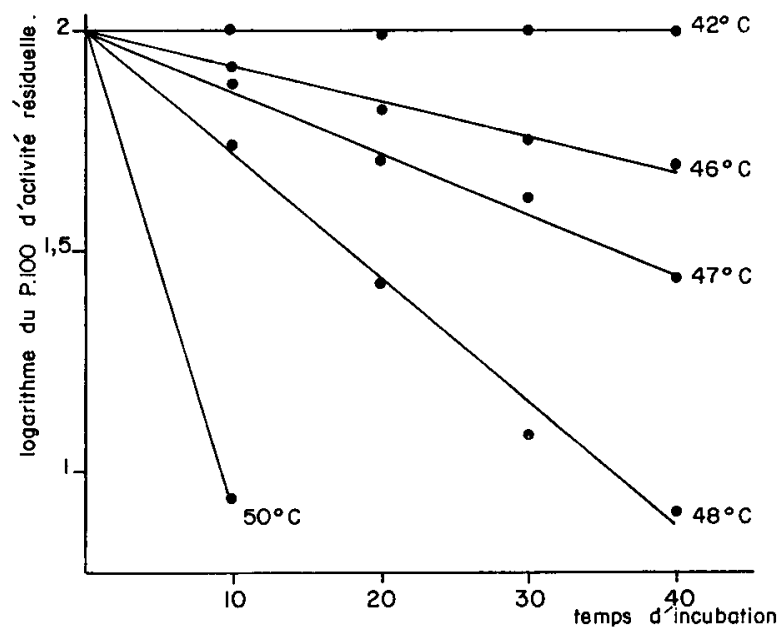

FIG. 7. - Stabilité ò différentes températures.

Inactivation en tampon acétate $0,1 \mathrm{M} \mathrm{pH} 5,5$.

Mélange d'hydrolyse : $-0,1 \mathrm{ml}$ de solution enzymatique inactivée ; $-0,9 \mathrm{ml}$ de Z-Glu-Tyr. $0,8 \mathrm{mM}$ en tampon acétate $0,1 \mathrm{M} \mathrm{pH} \mathrm{4,0}$.

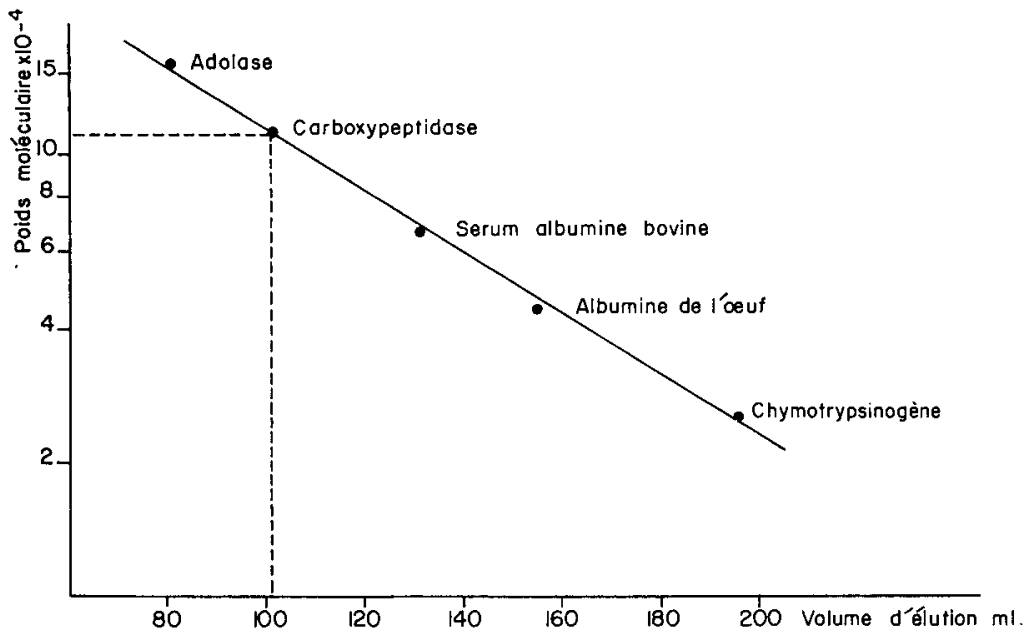

FIG. 8. - Estimation du poids moléculaire par filtration sur Bio-Gel P-150.

captoéthanol et le dithiothreitol. La carboxypeptidase a été soumise à l'action des réactifs des groupes $\mathrm{SH}\left(\mathrm{H}_{\mathrm{g}} \mathrm{Cl}_{2}, \mathrm{PMC}, \mathrm{MMB}\right.$, PHMB, MIAA) : l'inhibition quand elle est observée reste faible, l'activité résiduelle étant au moins égale à 80 p. 100. Si l'on fait varier les conditions d'incubation avec le PHMB ef le MIAA en modifiant le $\mathrm{pH}$ (de $\mathrm{pH} 4,5$ à 6,5 en tampon acétate ou phosphate) ou la température ( 4 ou $20^{\circ} \mathrm{C}$ ), on n'observe pas d'inhibition supérieure aux précédentes. Il est donc peu probable qu'un 
groupement $\mathrm{SH}$ soit impliqué dans la réaction. Les inhibiteurs de la trypsine (TLCK) et de la chymotrypsine (TPCK) n'ont pas d'action sur l'activité enzymatique. En revanche l'enzyme est inhibée par le DFP ce qui indique qu'un groupement sérine intervient dans la réaction. L'inhibition est fonction du $\mathrm{pH}$. Elle augmente entre $\mathrm{pH}$ 5,0 et 6,5 sans toutefois être complète pour cette dernière valeur (tabl. 2).

\section{TABLEAU 2}

Effet des inhibiteurs

(Activité résiduelle mesurée selon Matériel et Méthodes et exprimée en p. 100 par rapport à un témoin incubé sans inhibiteur dans les mêmes conditions)

\begin{tabular}{|c|c|c|c|c|c|}
\hline \multirow[b]{2}{*}{ Inhibiteur } & \multicolumn{4}{|c|}{ Conditions de pré-incubation } & \multirow{2}{*}{$\begin{array}{l}\text { Activité } \\
\text { résiduelle } \\
\text { (p. 100) }\end{array}$} \\
\hline & Molarifé & $\begin{array}{c}\text { Temps } \\
\text { (mn) }\end{array}$ & $\begin{array}{c}\text { Température } \\
\left({ }^{\circ} \mathrm{C}\right)\end{array}$ & Tampon $0,1 \mathrm{M}$ & \\
\hline 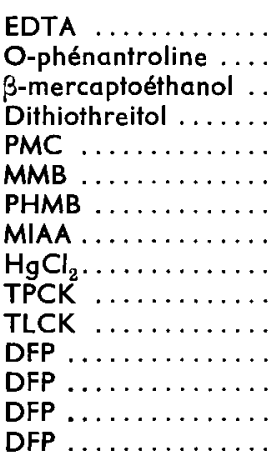 & $\begin{array}{r}10^{-3} \\
5.10^{-3} \\
10^{-3} \\
10^{-3} \\
10^{-3} \\
10^{-3} \\
10^{-3} \\
10^{-3} \\
10^{-3} \\
10^{-3} \\
10^{-3} \\
10^{-3} \\
10^{-2} \\
10^{-2} \\
10^{-2}\end{array}$ & $\begin{array}{r}30 \\
30 \\
30 \\
30 \\
30 \\
30 \\
30 \\
30 \\
30 \\
30 \\
30 \\
180 \\
180 \\
180 \\
180\end{array}$ & $\begin{array}{r}20 \\
20 \\
20 \\
20 \\
20 \\
20 \\
20 \\
20 \\
20 \\
20 \\
20 \\
4 \\
4 \\
4 \\
4\end{array}$ & $\begin{array}{l}\text { Acétate pH 5,5 } \\
\text { Acétate pH 5,5 } \\
\text { Acétate pH 5,5 } \\
\text { Acétate pH 5,5 } \\
\text { Acétate pH 5,5 } \\
\text { Acétate pH 5,5 } \\
\text { Acétate pH } 5,5 \\
\text { Acétate pH 5,5 } \\
\text { Acétate pH 5,5 } \\
\text { Acétate pH 5,5 } \\
\text { Acétate pH 5,5 } \\
\text { Phosphate pH 5,0 } \\
\text { Phosphate pH 5,0 } \\
\text { Phosphate pH 6,0 } \\
\text { Phosphate pH 6,5 }\end{array}$ & $\begin{array}{r}94 \\
104 \\
94 \\
79 \\
80 \\
100 \\
81 \\
106 \\
105 \\
93 \\
87 \\
87 \\
68 \\
35 \\
18\end{array}$ \\
\hline
\end{tabular}

\section{Action des cations métalliques.}

Les sels minéraux suivants : $\mathrm{ZnSO}_{4}, \mathrm{MgSO}_{4}, \mathrm{MnSO}_{4}, \mathrm{FeSO}_{4}, \mathrm{CuCl}_{2}, \mathrm{CoCl}_{2}$ ont été testés à la concentration de $10^{-3} \mathrm{M}$. Aucune influence notable n'a été observée ce qui suggère que l'activité enzymatique n'est pas affectée par les cations métalliques ou les ions $\mathrm{Cl}^{-}$ef $\mathrm{SO}_{4}^{--}$.

\section{Spécificité d'action.}

Parmi les peptides de synthèse testés seuls le Z-Glu-Tyr, le Z-Glu-Phe et le LeuLeu-Leu sont hydrolysés avec un bon rendement (tabl. 3). Contrairement à certaines carboxypeptidases d'A. oryzae (Nakadai, Nasuno ef Igushi, 1972a,b,c, 1973a) l'enzyme de $P$. roqueforti hydrolyse faiblement ou pas les substrats possédant une glycine en position pénultième, en particulier le Z-Gly-Phe est beauccup moins bien hydrolysé que le Z-Glu-Phe. De même les substrats ayant une glycine en position C-terminale sont faiblement hydrolysés. L'action sur les dipeptides est faible (Leu-Leu est beaucoup moins attaqué que le tripeptide Leu-Leu-Leu). 
TABLEAU 3

Hydrolyse des substrats synthétiques

\begin{tabular}{lcrc}
\hline Substrat & Activité & Substrat & Activité \\
\hline Z-Glu-Tyr & 0,600 & Z-Gly-Gly-Gly & 0,025 \\
Z-Glu-Phe & 0,580 & Leu-Leu-Leu & 0,520 \\
Z-Gly-Met & 0,115 & Leu-Gly-Gly & 0,055 \\
Z-Gly-Val & 0,090 & Met-Leu-Gly & 0,025 \\
Z-Gly-Phe & 0,040 & Gly-Try-Gly & 0,040 \\
Z-Gly-Arg & 0,030 & Gly-Tyr-Gly & 0,010 \\
Z-Gly-Ser & 0,020 & Leu-Leu & 0,045 \\
Z-Gly-X* & 0,000 & Leu-Tyr & 0,070 \\
Bz-Gly-Lys & 0,000 & Z-Gly-Phe-NH & 0,000 \\
& & Z-Gly-Val-NH., & 0,000 \\
\hline
\end{tabular}

*X : Asp, Pro, Gly, Ala, Try.

Mélange d'hydrolyse :

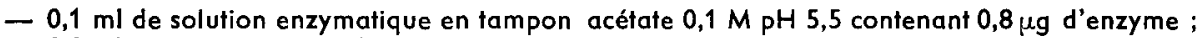
- $0,9 \mathrm{ml}$ de substrat $0,510^{-3} \mathrm{M}$ en tampon acétate $0,1 \mathrm{M} \mathrm{pH} 4,0$.

Incubation : 3 h à $35^{\circ} \mathrm{C}$.

Activité exprimée en différence de densité optique à $570 \mathrm{~nm}$ (après coloration à la ninhydrine) par rapport à un blanc incubé sans enzyme.

Après $24 \mathrm{~h}$ d'hydrolyse le glucagon est profondément hydrolysé (fig. 9). Du fait de la séparation incomplète des résidus Thr, Ser, Asn et Gln sur autoanalyseur et de la présence de résidus identiques en plusieurs points de la chaîne on ne peut préciser exactement où s'arrête l'hydrolyse. Parmi les résidus présents à un seul exemplaire dans la chaîne du glucagon ( $\mathrm{Met}_{27}, \mathrm{Val}_{23}, \mathrm{Ala}_{19}$, $\mathrm{Lys}_{12}$ ef $\mathrm{Gly}_{4}$ ) on constate que $\mathrm{Val}_{23}$, $\mathrm{Ala}_{19}$ et Lys ${ }_{12}$ représentent respectivement 88, 91 et 60 p. 100 de Met $_{27}$ et que Gly ${ }_{4}$ 'est pas détectée, ce qui implique que l'hydrolyse s'arrête entre Lys 1 $_{12}$ et Gly. II apparaît donc que la spécificité de l'enzyme est large puisqu'elle est capable de libérer comme la carboxypeptidase $A$ des acides aminés hydrophobes mais aussi des acides aminés acides et basiques (Asp, Lys et Arg).

Sur le substrat His-Ser-Gln-Gly-Thr-Phe (hexapeptide N-terminal du glucagon) on observe que le résidu $\mathrm{Gln}_{3}$ est libéré en faible quantité (fig. 9) et que le dipeptide $\mathrm{His}_{1}-\mathrm{Ser}_{2} \mathrm{n}^{\prime}$ est pas coupé. Dans la mesure où les résidus Gln sont facilement libérés dans le glucagon ( $\mathrm{Gl}_{24}$ ef $\mathrm{Gln}_{20}$ ) la faible libération de la glutamine dans le tripeptide His-Ser-Gln peut être imputée à un effet de longueur de chaîne, les petits substrats (tripeptides) étant moins bien hydrolysés que les substrats plus longs.

Sur la chaîne de l'insuline l'alanine est libérée rapidement et en grande quantité. Les acides aminés suivants sont présents dans l'hydrolysat mais en quantités trop faibles pour être mesurées. La liaison Lys-Pro est donc hydrolysée à une vitesse très faible ce qui empêche la coupure des liaisons suivantes. Dans la mesure où la lysine est bien libérée dans le glucagon (liaison $\mathrm{Ser}_{11}-$ Lys $_{12}$ ) on peut conclure que la proline est responsable du très faible rendement obtenu pour cette liaison. 


\section{GLUCAGON}

5 10

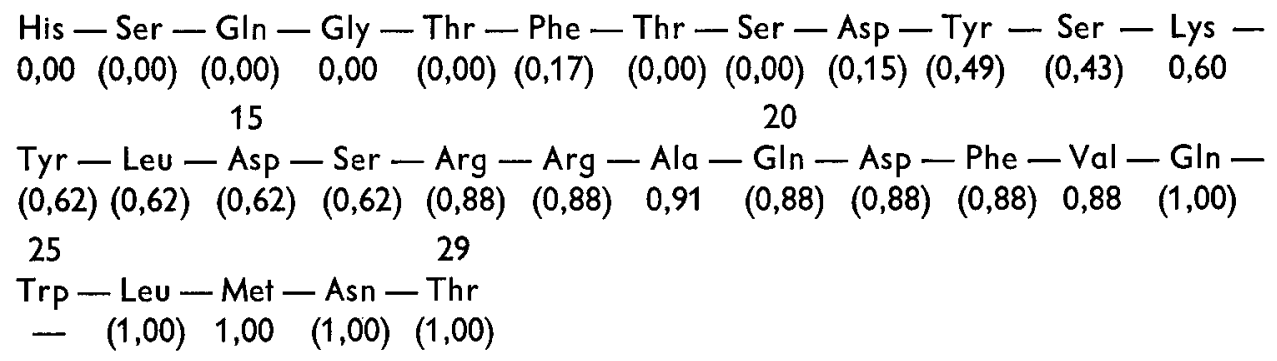

\section{HEXAPEPTIDE}

$\mathrm{His}$ - Ser - Gln - Gly - Thr - Phe

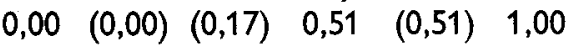

INSULINE

12025

Phe ----- Cys $\mathrm{SO}_{3} \mathrm{H}-\mathrm{Gly}$ - Glu - Arg - Gly - Phe - Phe - Tyr - Thr - Pro 30

Lys - Ala

$+\quad 1,00$

FIG. 9. - Hydrolyse des chaines peptidiques par lo carboxypeptidase.

Rapport molaire des acides aminés libérés. Les chiffres entre parenthèses représentent les résidus hydrolysés en plusieurs endroits de la chaîne ou bien insuffisamment séparés sur autoanalyseur (Ser, Thr, GIn, Asn). Les quantités ont été calculées en supposant que chaque résidu esf inférieur ou égal au précédent et supérieur ou égal au suivant.

+ : acide aminé présent en quantité trop faible pour être mesurée.

\section{Propriétés de la fraction B.}

La fraction $B$ est séparée de l'extrait purifié par chromatographie sur CM-cellulose et représente une faible partie (5 p. 100) de l'activité totale (voir purification). Elle a été comparée succinctement à la préparation purifiée. Les inhibitions par le PHMB et le DFP sont très comparables en intensité et, pour le DFP, présentent la même dépendance vis-d̀-vis $d u \mathrm{pH}$. Le poids moléculaire estimé par filtration sur BioGel P-150 dans les mêmes conditions que l'enzyme purifiée est de 105000 daltons. La fraction $B$ présentant donc des propriétés très comparables à la carboxypeptidase purifiée, son étude n'a pas été poursuivie. 


\section{Conclusion.}

Le glucagon a été utilisé comme substrat pour les carboxypeptidases de $P$. janthinellum (carboxypeptidases $S_{1}$ ) (Jones et Hofmann, 1972), d'A. saitoi (Ichishima et Arai, 1973), d'une levure (carboxypeptidase Y, Hayashi, Moore et Stein, 1973a) et de la graine de coton (Ihle et Dure, 1972). Pour toutes ces enzymes ce substrat est profondément dégradé puisque l'on observe la libération d'acides aminés correspondant à l'hydrolyse de 9 liaisons pour la carboxypeptidase $Y, 12$ liaisons pour la carboxypeptidase d'A. saitoi et 19 liaisons pour la pénicillo-carboxypeptidase $S_{1}$. L'enzyme de $P$. roqueforti coupe pour le moins 18 acides aminés sur cette chaîne protéique et présente donc une spécificité large comme les carboxypeptidases précédentes (libération des acides aminés basiques acides et neutres). Sur la chaîne B de l'insuline l'hydrolyse de la liaison $\mathrm{PrO}_{28}-\mathrm{Lys}_{29}$ est très lente alors que celle-ci est très bien coupée par la carboxypeptidase Y (Hayashi, Moore et Stein, 1973a) et la pénicillocarboxypeptidase $S_{1}$ (Jones et Hofmann, 1972). Toutefois pour cette dernière il a été montré (Hui et al., 1974) que dans certains cas la proline en position pénultième éłait un obstacle à I'hydrolyse (les liaisons Pro-Ala, Pro-Gly, Pro-Ser et Pro-Thr sont faiblement ou pas coupées).

Les peptides synthétiques contenant une glycine en position C-terminale ou $C$-pénultième sont peu hydrolysées par la carboxypeptidase de $P$. roqueforti. Là encore un comportement analogue a été observé pour les carboxypeptidases de A. saitoi (Ichishima, 1972), de la feville d'oranger (Sprossler ef al., 1971) et la carboxypeptidase $Y$ (Hayashi, Moore et Stein, 1973a). Toutefois certains de ces substrats sont bien hydrolysés par les carboxypeptidases d'A. oryzae (Nakadai, Nasuno, Igushi, 1972a,b,c, 1973a).

L'enzyme de $P$. roqueforti est inhibée par le DFP, il est donc probable qu'un résidu sérine intervient dans la réaction d'hydrolyse. Pour la carboxypeptidase $Y$ (Hayashi, Moore et Stein, 1973b) et une carboxypeptidase isolée du haricot (Shaw ef Wells, 1972) (qui sont également inhibées par le DFP), on a pu isoler la séquence contenant la sérine impliquée. Cette séquence est identique pour les deux enzymes et diffère de celle des sérines protéases (protéases alcalines) déjà étudiées. L'inhibition de l'enzyme de $P$. roqueforti est fonction du $\mathrm{pH}$, une concentration de $10^{-2} \mathrm{M}$ et un $\mathrm{pH}$ de 6,5 étant les conditions les plus favorables que nous ayons testées.

De par l'ensemble des propriétés précédentes il apparaît nettement que l'enzyme de $P$. roqueforti appartient au groupe des carboxypeptidases acides (caractérisées par une spécificité large ef l'inhibition par les réactifs de la sérine) mis en évidence non seulement chez les levures et moisissures (Hayashi, Moore et Stein, 1973a ; Nakadai, Nasuno et Igushi, 1972a; Jones ef Hofmann, 1972 ; lehishima, 1972) mais aussi chez certains végétaux supérieurs (Visuki, Mikoba et Enari, 1969; Kubola et al., 1973 ; Sprossler, 1971, Ihle ef Dure, 1972). Pour plusieurs de ces enzymes (Hayashi, Moore et Stein, 1973b ; Nakadai, Nasuno ef Igushi, 1972a ; Jones ef Hofmann, 1972 ; Ichishima, 1972 ; Visuki, Mikoba et Enari, 1969) l'intervention d'un groupe SH dans la réaction a été suggérée car elles sont inhibées par les réactifs classiques de ces groupements. L'enzyme de $P$. roqueforti est inhibée très partiellement par ces réactifs. Dans 
la mesure où ces inhibiteurs sont des petites molécules, de dimensions comparables à celles des substrats, il semble peu probable que l'absence d'inhibition forte soit due à un empêchement stérique. De plus le chlorure de mercure n'est lui-même pas inhibiteur. Il semble donc qu'il n'y a pas de groupe SH impliqué directement dans le mécanisme d'action. Notons que les carboxypeptidases isolées de l'exocarpe du citron (Kubota et al., 1973) et du fruit de la tomate (Matoba et Doi, 1974), présentent vis-à-vis du MIAA et du PHMB un comportement comparable à l'enzyme de $P$. roqueforti.

La quantité d'enzyme produite dans le milieu après 3 jours de culture (c'est-à-dire au moment de la croissance maximale) est faible $(0,7 \mathrm{mg} / \mathrm{l})$ et correspond à une activité carboxypeptidasique nettement inférieure à celles détectées par Yokoyama et al. (1974) pour d'autres espèces de Penicillium. La poursuite des cultures jusqu'à 6 jours en milieu à $\mathrm{pH}$ contrôlé $(\mathrm{pH}=5)$ entraîne une lyse notable du mycelium sans augmenter l'activité produite dans le milieu. L'enzyme serait donc produite avant la lyse du mycelium et serait de nature exocellulaire.

La synthèse de plusieurs carboxypeptidases acides a été montrée pour plusieurs moisissures : chez $P$. janthinellum deux carboxypeptidases sont sécrétées (Jones et Hofmann, 1972), chez A. oryzae on a conclu à l'existence de 4 carboxypeptidases acides (Nakadai, Nasuno ef Iguchi, 1972a,b,c, 1973). Chez $P$. roqueforti, deux fractions carboxypeptidasiques sont séparées par chromatographie sur CM-cellulose. Toutefois une comparaison des deux préparations montre qu'elles ont des propriétés voisines. La différence de comportement chromatographique peut traduire la synthèse de deux enzymes aux propriétés proches comme dans le cas d'A. oryzae. A l'inverse on peut supposer que $P$. roqueforti synthétise une seule carboxypeptidase acide et que la présence de deux fractions est due à une protéolyse partielle de l'enzyme par la protéase acide au cours de la production ou des premiers stades de purification.

Accepté en novembre 1976.

Remerciements. - Nous tenons à remercier Madame Ghislaine Brignon et Monsieur B. Ribadeau Dumas qui ont effectué les analyses d'acides aminés ainsi que Monsieur J. Hermier pour ses suggestions au cours de la réalisation de ce travail et de la rédaction du manuscrit.

\section{Références}

ANDREWS P., 1964. Estimation of the molecular weights of proteins by Sephadex gel-filtration. Biochem. J., 91, 222-233.

ARAI T., ICHISHIMA E., 1974. Mode of action on protein of acid carboxypeptidase from Aspergillus saifoi. J. Biochem., 76, 765-769.

CHRAMBACH A., REISFELD R. A., WYCKOFF, M., ZACCARI, J., 1967. A procedure for rapid and sensitive staining of protein fractionated by polyacrylamide gel electrophpresis. Anal. Biochem. 20, $150-154$.

DUNKER A. K., RUECKERT R. R., 1969. Observations on molecular weight determination on polyacrylamide gel. J. biol. Chem., 244, 5074-5080.

FUKUMOTO J., TSURU D., YAMAMOTO T., 1967. Studies on mold protease. I. Purification, crystallization and some enzymatic properties of acid protease of Rhizopus chinensis. Agr. Biol. Chem., 31, 710-717. 
GRIPON J.-C., HERMIER J., 1974. Le système protéolytique de Penicillium roqueforti. IlI. Purification, propriétés et spécificité d'une protéase inhibée par I'E.D.T.A. Biochimie, 56, 1323-1332.

HAYASHI K., FUKUSHIMA D., MOJI K., 1967. Isolation of alkaline proteinase from Aspergillus sojoe in homogeneous form. Agr. Biol. Chem., 31, 1237-1241.

HAYASHI R., MOORE S., STEIN W. H., 1973a. Carboxypeptidase from yeast. Large scale preparation and the application to COOH-terminal analysis of peptides and proteins. J. biol. Chem., 248, 2296-2302.

HAYASHI R., MOORE S., STEIN W. H., 1973b. Serine at the active center of yeast carboxypeptidase. J. biol. Chem., 248, 8366-8369.

HOFMANN T., SHAW R., 1964. Proteolytic enzymes of Penicillium janthinellum. I. Purification and properties of a trypsinogen-activating enzyme (peptidase A). Biochem. Biophys. Acta, 92, 543-557.

HUI A., RAO L., KUROSKY A., JONES S. R., MAINS G., DIXON J. W., SZEWEZUK A., HOFMANN T., 1974. The use of penicillocarboxypeptidase. $S_{1}$ in amino acid sequencing. Arch. Biochem. Biophys., 160, 577-587.

ICHISHIMA E., 1972. Purification and characterisation of a new type of acid carboxypeptidase from Aspergillus. Biochem. Biophys. Acta, 258, 274-288.

ICHISHIMA E., ARAI T., 1973. Specificity and mode of action of acid carboxypeptidase from Aspergillus saifoi. Biochem. Biophys. Acto, 293, 444-450.

IHLE J. N., DURE L. S., 1972. The developmental biochemistry of cottonseed embryogenesis and germination. II. Catalytic properties of the cotton carboxypeptidase. J. biol. Chem., 247, 5041-5047.

JONES S. R., HOFMANN T., 1972. Penicillocarboxypeptidase-S, a non-specific SH-dependent exopeptidase. Can. J. Biochem., 50, 1297-1310.

KUBOTA Y., SHOJ S., FUNAKOSHI T., UEKI H., 1973. Carboxypeptidase $C_{N}$. I. Purification and characterization of the enzyme from the exocarp of Citrus natsudaidai Hayata. J. Biochem., 74, 757-770.

LOWRY O. H., ROSEBROUGH N. J., FARR A. L., RANDALL R. J., 1951. Protein measurement with the Folin phenol reagent. J. biol. Chem., 193, 265-275.

MATOBA T. ef DOI E., 1974. Carboxypeptidase activity of tomato fruit during the ripening process and some enzymatic properties. Agr. Biol. Chem., 38, 1901-1905.

MOELWYN-HUGHES E. A., 1950. The Enzymes, vol. 1, pp. 28-78, SOMNER J. B., MYRBACH K., Ed. Academic Press, New York.

MOORE S., STEIN W. H., 1954. A modified ninhydrin reagent of the photometric determination of amino-acids and related compounds. J. biol. Chem., 211, 907-913.

NAKADAI T., NASUNO S., IGUGHI N., 19720. Purification and properties of acid carboxypeptidase I from Aspergillus oryzae. Agr. Biol. Chem., 36, 1343-1352.

NAKADAI T., NASUNO S., IGUCHI N., 1972b. Purification and properties of acid carboxypeptidase Il from Aspergillus oryzae. Agr. Biol. Chem., 36, 1473-1480.

NAKADAI T., NASUNO S., IGUCHI N., 1972c. Purification and properties of acid carboxypeptidase III from Aspergillus oryzoe. Agr. Biol. Chem., 36, 1481-1488.

NAKADAI T., NASUNO S., IGUCHI N., 1973a. Purification and properties of acid carboxypeptidase IV from Aspergillus oryzae. Agr. Biol. Chem., 37, 1237-1251.

NAKADAI T., NASUNO S., IGUCHI N., 1973b. Purification and properties of neutral proteinase I from Aspergillus oryzae. Agr. Biol. Chem., 37, 2695-2701.

REISFELD R. A., LEWIS U. J., WILLIAMS D. E., 1962. Disc electrophoresis of basic proteins and peptides on polacrylamide gels. Nature, 195, 281-283.

SEKINE H., 1972. Neutral proteinases I ef II of Aspergillus sojoe. isolation in homogeneous form. Agr. Biol. Chem., 36, 198-206. 
SHAW D. C., WELLS J. R. E., 1972. Radiochemical determination of a unique sequence around the reactive serine residue of a di-isopropyl phosphorofluoridate-sensitive plant carboxypeplidase and a yeast peptidase. Biochem. J., 128, 8366-8369.

SPROSSLER B., HEILMANN H. D., GRAMPP E., UHLIG H., 1971. Eigenschaften der carboxypeptidase $C$ aus orengenblättern. Hoppe-Seyler's Z. Physiol. Chem., 352, 1524-1530.

TURKOVA J., MIKES O., GANGEV K., BOUBLIK M., 1969. Isolation and characterization of alkaline proteinase of Aspergillus flavus. Biochem. Biophys. Acta, 178, 100-111.

URIEL J., 1966. Méthode d'électrophorèse dans les gels d'acrylamide agarose. Bull. Soc. Chim. Biol., 48, 969-982.

VISUKI K., MIKOBA J., ENARI T. M., 1969. Isolation and partial characterization of a carboxypeptidase from barley. Eur. J. Biochem., 7, 193-199.

YOKOGAMA S., OOBAYASHI A., TANABE O., SUGAWARA S., ARAKI E., ICHISHIMA E., $1974 . \quad$ Production and some properties of a new type of acid carboxypeptidase of Penicillium mold. Appl. Microb., 27, 953-960.

YU J., IWASAKI S., TAMURA G., ARIMA K., 1968. Physical properties and amino acid composition of Mucor rennin crystal isolated from Mucor pusillus var. Lindf. Agr. Biol. Chem., 32, 1051-1052. 